\title{
X-ray studies of solar system objects: past, present and the next decade
}

\author{
G. Branduardi-Raymont ${ }^{1, \star}$ \\ Mullard Space Science Laboratory, University College London, Holmbury St Mary, Dorking, Surrey, RH5 6NT, UK
}

Received 3 Oct 2016, accepted ?? 2016

Published online ??

Key words Solar system: general - X-rays: individual (solar system targets)

This paper presents an overview of the remarkable progress that X-ray studies of solar system objects have delivered over the past three decades, covering research that encompasses remote observations with XMM-Newton and Chandra, in situ measurements by planetary missions and models of solar wind propagation from 1 AU. The future of X-ray exploration of the solar system in the next decade is bright, counting on the continuing excellent imaging and spectral performance of XMM-Newton instruments, which is most effective when combined with the outstanding spatial resolution of Chandra and in particular while planetary missions are making in situ measurements of solar wind and local conditions.

(C) 2006 WILEY-VCH Verlag GmbH \& Co. KGaA, Weinheim

\section{Introduction}

Much of the progress in the X-ray exploration of solar system objects has been enabled by the XMM-Newton observatory, with its large effective area, the combination of medium and high X-ray spectral resolution afforded by the EPIC and RGS instruments and the availability of opti$\mathrm{cal} / \mathrm{UV}$ monitoring, for comets in particular. The complementarity of XMM-Newton with the Chandra observatory, which offers exquisite spatial resolution although at lower sensitivity, has led and is leading to giant steps in furthering our understanding of the physics underpinning planetary Xray emissions. Moreover, the combination of remote observations and in situ measurements (or the use of solar wind parameter propagations from $1 \mathrm{AU}$ ) have demonstrated the importance of obtaining deeper insights in the mechanisms linking the X-ray emission with the local plasma and magnetic field conditions. In the following sections the achievements made and the knowledge derived from past and current observations of solar system targets are reviewed and the aspirations for further research in this field in the next decade are discussed.

\section{Early studies of Jupiter, charge exchange and comets}

Jupiter and its satellite system have been the target of the vast majority of planetary X-ray measurements so far; much has been revealed of the extreme physical conditions existing in their environment, and new questions have been raised. The first detection of Jupiter with the Einstein Observatory IPC and HRI (Metzger et al. 1983) marked the beginning of X-ray observations of the solar system. Soon

\footnotetext{
^ Corresponding author: e-mail: g.branduardi-raymont@ucl.ac.uk
}

the emission was associated with heavy ion precipitation and charge transfer (Waite et al. 1988, Horanyi, Cravens \& Waite 1988). The charge transfer hypothesis was clinched by the ROSAT discovery of X-rays from comet Hyakutake (Lisse et al. 1996): this was interpreted as charge transfer of solar wind heavy ions (Cravens 1997), later leading to what we now call Charge eXchange (CX) to be firmly established as the X-ray emission mechanism (for a detailed review of this process see Dennerl 2010). When CX takes place during the encounter of a solar wind (SW) heavy ion with a neutral particle the process takes the name of Solar Wind Charge eXchange (SWCX). The SWCX emissivity can be represented as $P_{X} \propto n_{N} n_{S W} v_{S W}$ (Cravens 2000) where $n_{N}$ is the local density of neutrals, and $n_{S W}$ and $v_{S W}$ represent the density of the solar wind and its velocity, respectively. Comets are spectacular sources of soft $\mathrm{X}$-ray emission because of the extended nature of their neutral comae where SWCX can take place. Fig. 1 shows the XMM-Newton EPIC image of comet C/2000 WM1 in Dec. 2001 (Dennerl et al. 2003): the emission practically fills the whole $30^{\prime}$ wide field-of-view, extending far more than the optical coma, and displays the curvature typical of a bow shock.

Cometary spectra are characterised by a wealth of emission lines, corresponding to the heavy ion species present in the solar wind (Bodewits et al. 2007). Returning to Jupiter, the first Chandra observation of the planet (Gladstone et al. 2002) revealed very unexpected results: soft $X$-rays are concentrated in a hot spot which is significantly pole-ward of latitudes magnetically connected with the inner magnetosphere, implying the ions are travelling in from as far out as 30 Jupiter radii ( $\mathrm{R}_{J}$; Fig. 2).

This raised the fundamental question, still debated today, of the origin of the ions: inner magnetosphere or solar wind? In the former case we would expect spectral signatures of oxygen and sulfur ions from Io's volcanoes, in the 


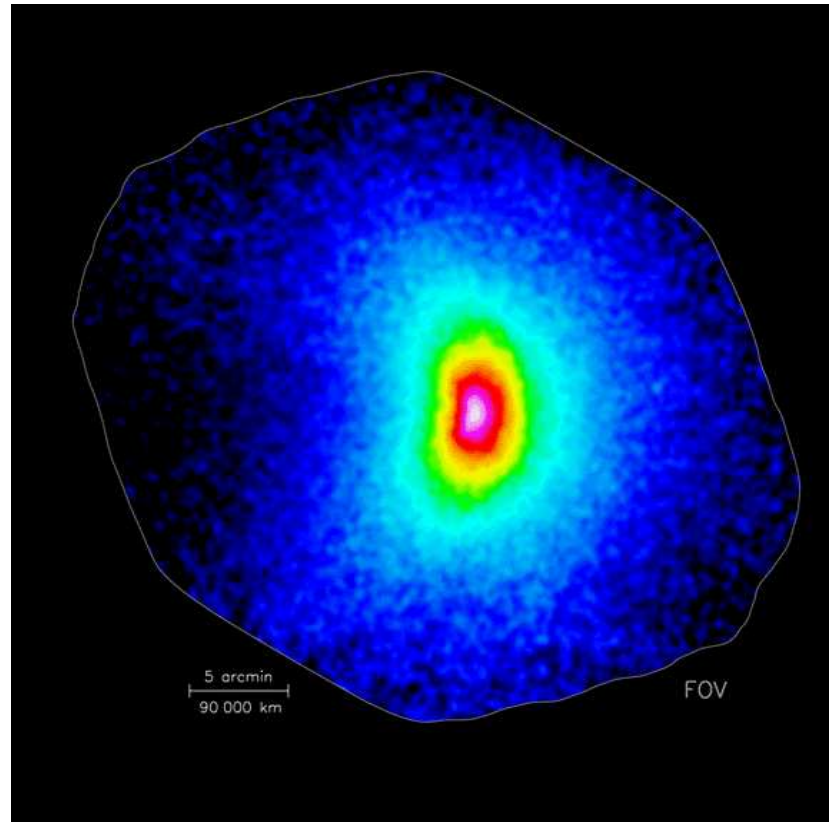

Fig. 1 Comet C/2000 WM1 as seen in the XMM-Newton EPIC camera in Dec. 2001. The soft X-ray emission produced by SWCX fills the detector field-of-view (Dennerl et al. 2003)

latter those of carbon ions, more abundant in the solar wind. Unfortunately the spectral resolution of XMM-Newton and Chandra CCDs is not sufficient to resolve the respective emission lines and the RGS has not sufficient sensitivity to separate the carbon and sulfur lines at its long wavelength end. Much modelling work (e.g. Hui et al. 2009, 2010 and references therein) has been carried out over the years in order to interpret the wealth of observational data, and in particular to reach a resolution of the question of the origin of the ions. While the models tend to prefer a magnetospheric origin, recent evidence (see below) suggests that the solar wind indeed plays a part in producing Jupiter's soft Xray aurora. The question then turns to what are the relative contributions of magnetospheric and solar wind ions to the emission.

Another surprising result from the Chandra observations of Gladstone et al. (2002) was the discovery of a strong pulsation in the hot spot X-rays with a period of $45 \mathrm{~min}$. Quasi-periodic variability and oscillations of differing duration and strength have been seen in later observations; such behaviour has been related to e.g. bounce periodicities in the magnetospheric scenario described by Cravens et al. (2003) or day-side pulsed reconnection, as modelled by Bunce et al. (2004).

Early results from Chandra also include the X-ray detections of the Galilean satellites Io and Europa, and of the Io plasma torus (Elsner et al. 2002; Fig. 3). While the emission from the satellites is attributed to fluorescence following energetic hydrogen, oxygen and sulfur ion impacts, that from the Io plasma torus is more puzzling, displaying a very soft continuum spectrum (which could be due to electron

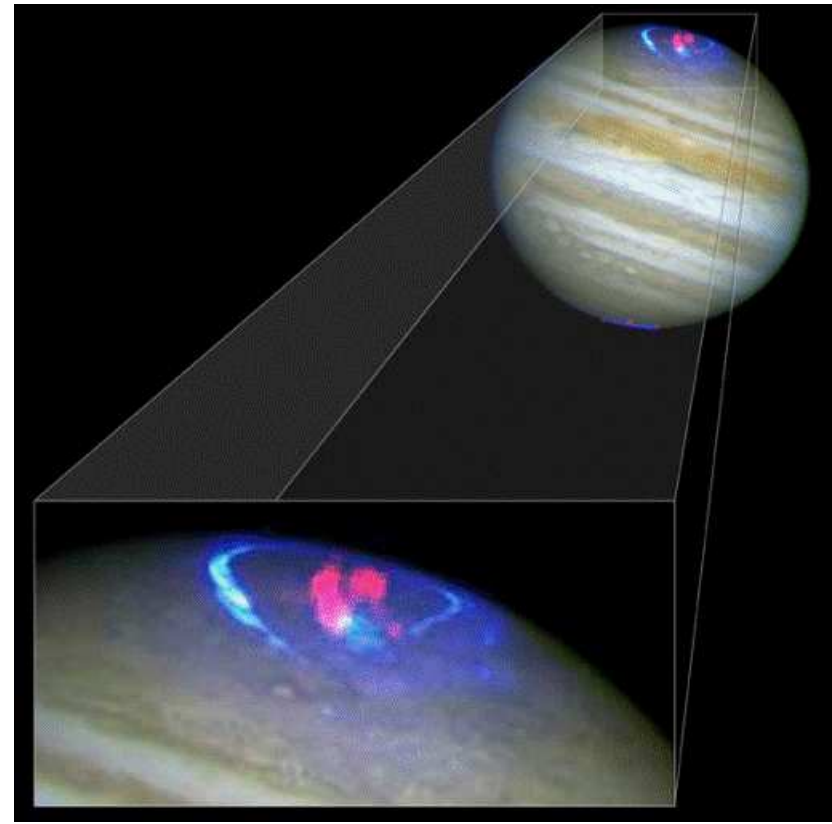

Fig. 2 Superposition of Chandra X-ray data (pink), HST STIS UV (blue) and HST optical (planet view). Credit NASA/CXC/SAO (chandra.harvard.edu/photo/2002/ 0001/more.html)

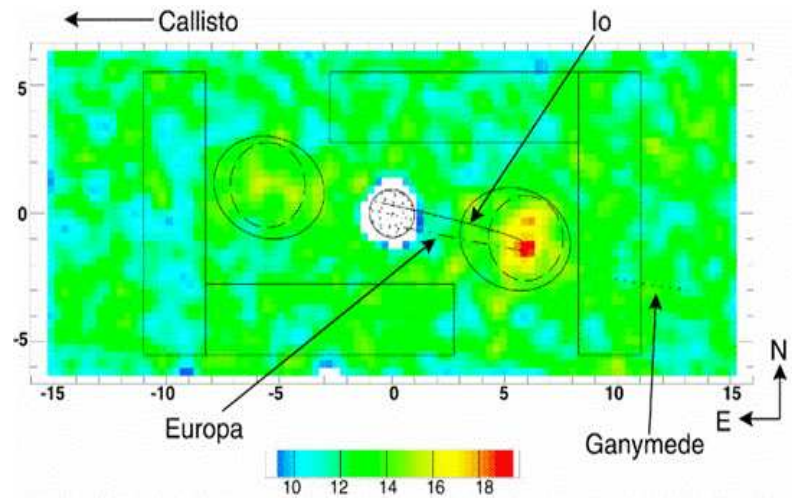

Fig. 3 Chandra ACIS image of the Io Plasma Torus (Elsner et al. 2002).

bremsstrahlung) and a strong and broad oxygen line, still unexplained.

XMM-Newton spectral maps (Fig. 4) clearly reveal the auroral soft X-ray ionic emissions in the narrow energy band centred on the OVII CX line (top left panel); unlike this, emission in the band centred on the FeXVII coronal line (top right panel), produced in the scattering of solar $\mathrm{X}$-rays by Jupiter's upper atmosphere, displays the morphology of the planetary disk. Surprisingly, auroral emission is also observed at higher energies (Fig. 4 bottom panels; the disk emission has practically disappeared at these energies) and its featureless continuum shape indicates it is due to bremsstrahlung by precipitating electrons of energies of few tens of $\mathrm{keV}$. This continuum was seen to vary drastically in shape and intensity between two consecutive XMM-Newton orbits (2-day long) in Nov. 2003 

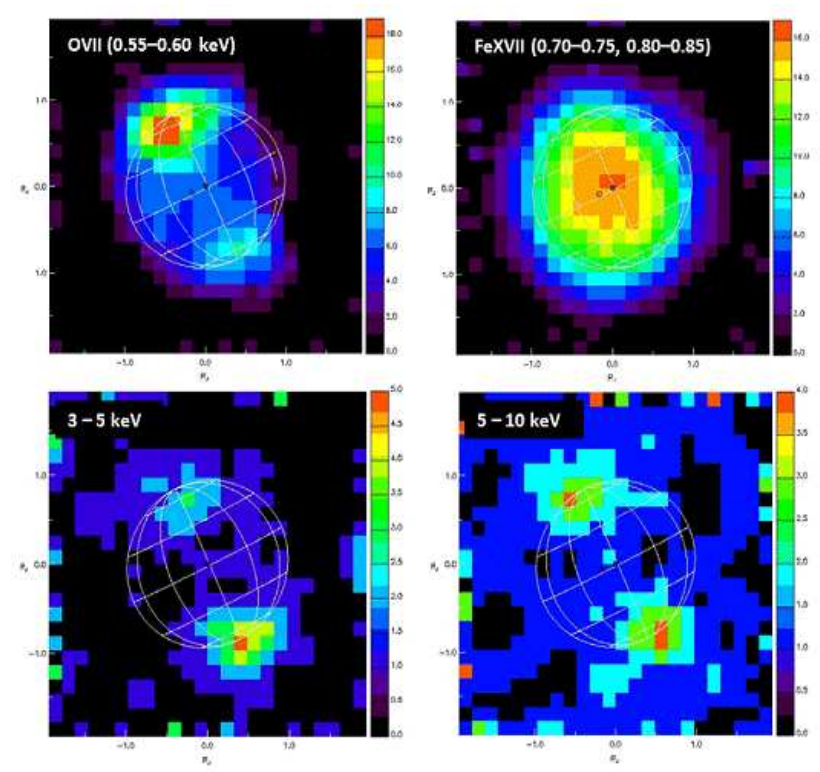

Fig. 4 XMM-Newton spectral maps of Jupiter's aurorae:at $<3$ $\mathrm{keV}$ ion emission is concentrated in the aurorae while the Jovian disk shines by scattering of solar X-rays (top panels); at $>3 \mathrm{keV}$ only the aurorae emit X-rays via electron bremsstrahlung (bottom) (Branduardi-Raymont et al. 2007).

(Branduardi-Raymont et al. 2007), around the time of the predicted arrival of the powerful Halloween solar wind storm.

Even more surprisingly, Chandra shows different spatial distributions for the low and high energy auroral emissions: while ionic $\mathrm{CX}$ soft $\mathrm{X}$-rays $(<2 \mathrm{keV})$ are concentrated in the hot spot, the harder $(>2 \mathrm{keV})$ electron bremsstrahlung X-ray events coincide with the FUV auroral oval (Fig. 5). Since the FUV emission is believed to be produced in the excitation of atmospheric $\mathrm{H}_{2}$ and $\mathrm{H}$ by $10-100 \mathrm{keV}$ electrons, the conclusion is that the same population of energetic electrons is likely to be responsible for both, the FUV and $\mathrm{X}$-ray emissions (Branduardi-Raymont et al. 2008).

XMM-Newton RGS data clearly resolve the auroral CX soft X-ray lines from those of the coronal-type scattered disk radiation, also revealing broad components in the profiles of the OVII and OVIII lines. Such broadening corresponds to velocities of $\pm 5000 \mathrm{~km} \mathrm{~s}^{-1}$ or energies of few $\mathrm{MeV}$ for oxygen ions, in line with model predictions (Branduardi-Raymont et al. 2007).

\section{Recent work on Jupiter}

More recently observations of Jupiter attempting to answer the question of the origin of the CX ions have used a different strategy, i.e. selecting times when a solar wind enhancement is expected to reach the planet, and looking for correlated enhancements in the X-ray emission. This worked well in Oct. 2011, when a target of opportunity observation was triggered with Chandra on the basis of propagation of solar wind parameters from $1 \mathrm{AU}$ to Jupiter. In this case an

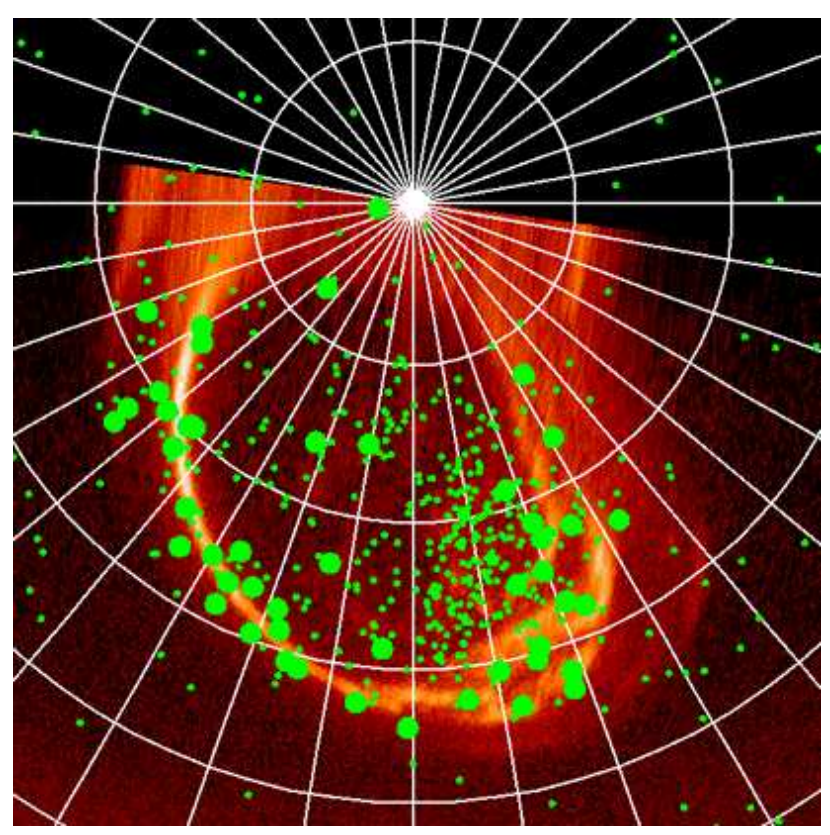

Fig. 5 Chandra X-ray events (small green dots: $<2 \mathrm{keV}$; large dots: $>2 \mathrm{keV}$ ) overplotted on an FUV image of Jupiter's North aurora taken simultaneously with HST (orange) (BranduardiRaymont et al. 2008).

8 -fold increase in the brightness of the soft X-ray aurora, coincident with the expected arrival of a coronal mass ejection, was observed between two observations separated by a couple of days (Fig. 6, Dunn et al. 2016).

Comparison of the morphological distribution of the Chandra X-ray events with the most recent model maps of the Jovian magnetic field (Vogt et al. 2011) indicates that $\mathrm{X}$-ray events fall consistently in the open-close field region of the magnetic footprints, suggesting dayside reconnection and possibly some penetration of the solar wind. Interestingly, prominent periodicities of 12 and 26 min were detected during the auroral enhancement in the first observation, and a weaker $\sim 45$ min period during solar wind quiescent conditions in the second. A similar search for Jovian auroral behaviour correlated with solar wind conditions was conducted during the large multi-wavelength observing campaign coordinated with the Hisaki satellite EUV spectroscopy run in 2014: also in this case the auroral hot spot events are seen to map near the magnetopause, half and half to close and open field line footprints respectively (Kimura et al. 2016).

We are now in a golden age for Jupiter exploration after the arrival of Juno at the planet in July 2016. XMMNewton and Chandra observations were carried out in May 2016 while Juno was in the solar wind before orbit injection; the aim was once again to correlate the X-ray auroral response to solar wind conditions, measured in situ by Juno, and hence much more reliable than those from propagations of measurements at $1 \mathrm{AU}$. These data are currently being analysed. 


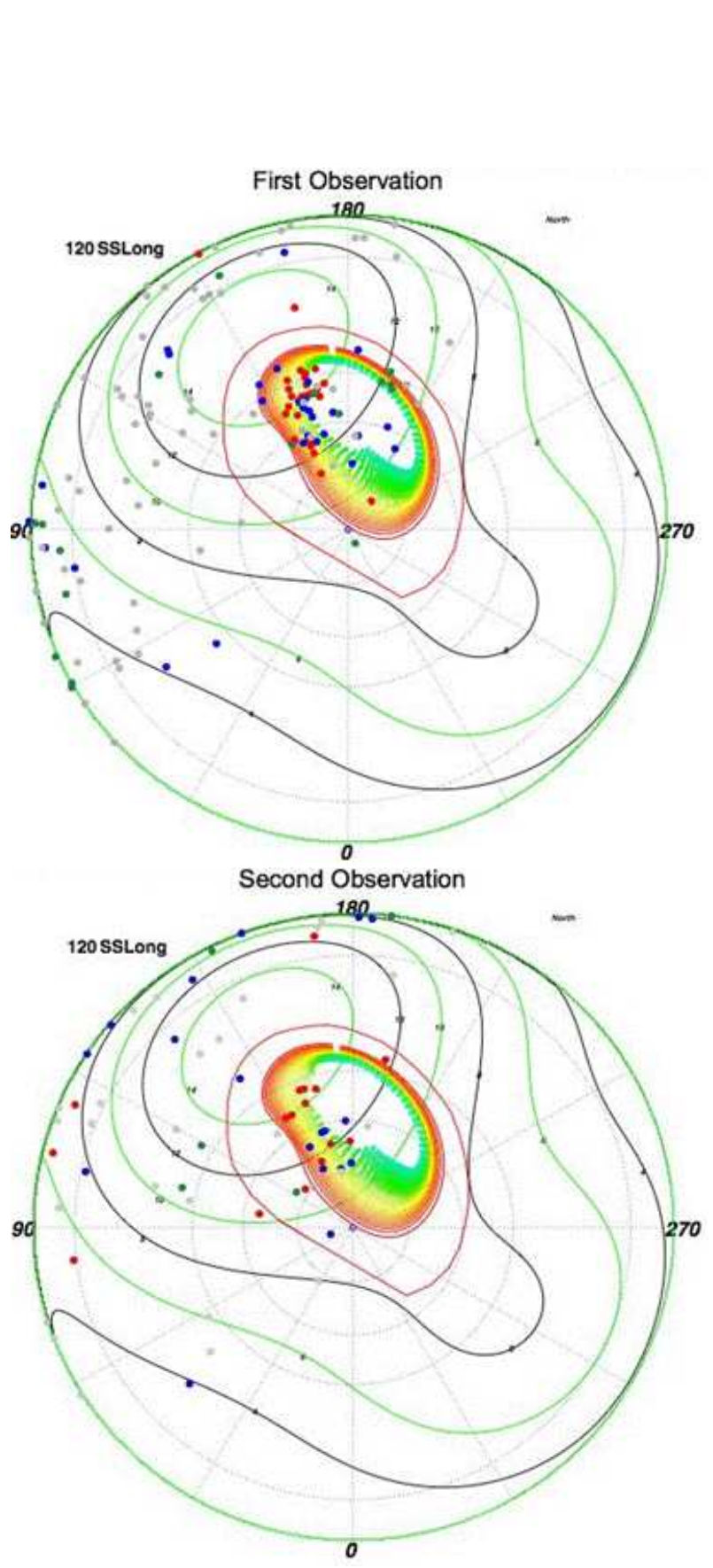

Fig. 6 Polar projections of two Chandra observations of Jupiter in Oct. 2011, separated by a couple of days (from Dunn et al. 2016). The first observation (top) is thought to coincide with the arrival of a coronal mass ejection at the planet, while the second (bottom) corresponds to quieter solar wind condition. Chandra X-ray events are colour coded: Red, 0.2-0.5 keV (carbon/sulfur); Blue, 0.5-0.8 keV (oxygen); Grey, 0.8-1.5 keV (solar); Green, > $1.5 \mathrm{keV}$. The coloured contours are from the most recent magnetic field model (Vogt et al. 2011) and represent the footprints of closed field lines mapping (in increments of $5 \mathrm{R}_{J}$ ) to $15-45 \mathrm{R}_{J}$ (red), $50-80 \mathrm{R}_{J}$ (yellow) and 85-150 $\mathrm{R}_{J}$ (green).

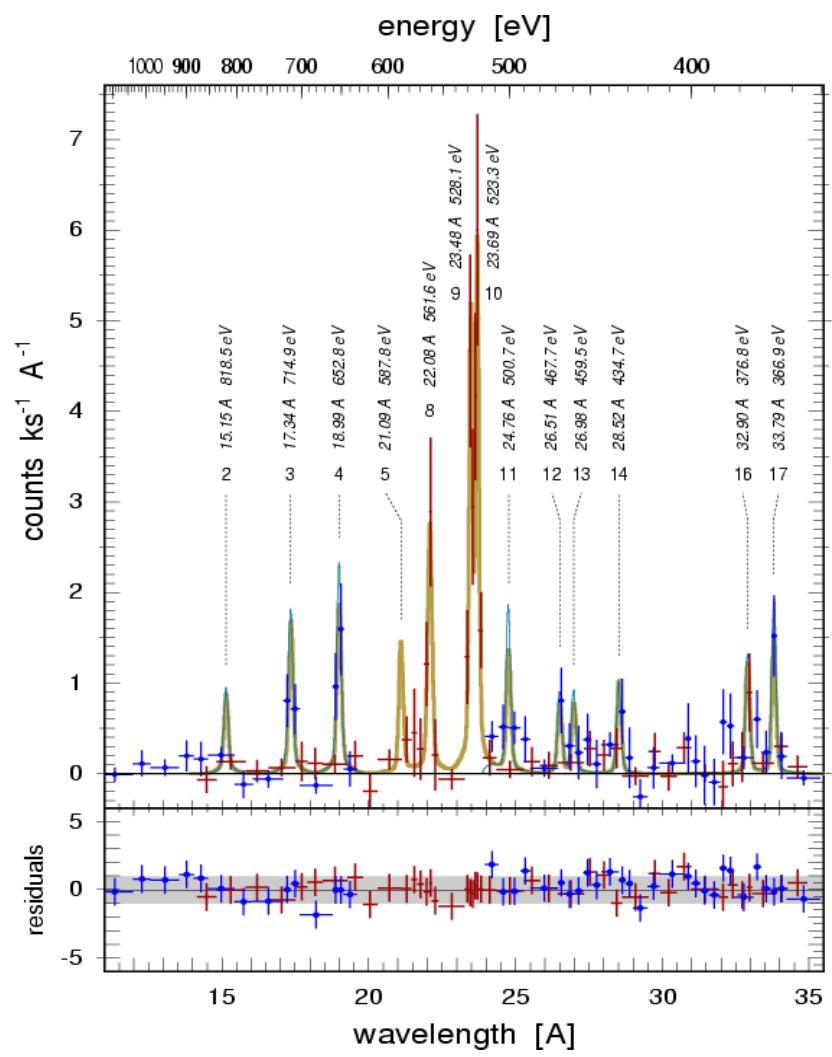

Fig. 7 XMM-Newton RGS spectrum of Mars extracted from a region $\pm 50^{\prime \prime}$ of the planet's centre in the cross dispersion direction, showing a mixture of SWCX and fluorescent scattering lines (such as the strong oxygen doublet at $\sim 23 \AA$ from scattering of solar X-rays in the planet's $\mathrm{CO}_{2}$ atmosphere).

\section{Saturn}

Unlike Jupiter, Saturn displays similar coronal type X-ray spectra from its disk and polar caps; the X-ray flux variability suggests that the emission is directly controlled by the Sun, on both short and long timescales, via elastic and fluorescent scattering of solar X-rays (Bhardwaj et al. 2005a). $\mathrm{X}$-ray emission, concentrated in an oxygen $\mathrm{K}_{\alpha}$ emission line, is also observed from the East ansa of its rings (Ness et al. 2004, Bhardwaj et al. 2005b), possibly a consequence of scattering of solar X-rays on atomic oxygen in the icy water ring material.

\section{Mars and Venus}

Turning to the un-magnetised planets in the solar system, in Nov. 2003 Mars provided a remarkable XMM-Newton RGS spectrum (Fig. 7, Dennerl et al. 2006), comprising, and allowing clear separation of, SWCX emission from its extended outflowing exosphere and fluorescent scattering of solar X-rays in its $\mathrm{CO}_{2}$ atmosphere. Separation of the two emission components was achieved by spatially selecting the expected regions of origin in the RGS cross dispersion direction: distances $|y| \leq 10^{\prime \prime}$ from the planet's centre for the 
disk emission, and two bands at $15^{\prime \prime}<|\mathrm{y}|<50^{\prime \prime}$ for the exosphere SWCX emission. Since the two components emit lines at different wavelengths, any (small) contamination between the two is eliminated by making use of the RGS high spectral dispersion. Such a wealth of emission lines has not been observed since (Dennerl, private communication) and clearly highlights the connection between the strength of the planetary X-ray emission and the high solar activity (Halloween storm) at the time of the 2003 observation.

Venus presents a similar case to Mars, combining exospheric SWCX emission with solar X-ray scattering in its dense atmosphere. The smaller scale height of its exosphere compared to Mars, though, makes detection of SWCX Xrays particularly difficult at times of high activity in the solar cycle, so that a definitive identification of the SWCX emission component has only been possible at solar minimum (Dennerl 2008).

\section{Earth}

The terrestrial soft X-ray aurora has been shown by Chandra HRC to be highly variable and to display a varied morphology of patches and arc-like structures (Bhardwaj et al. 2007). The emission is attributed to electron bremsstrahlung and K-shell lines of nitrogen and oxygen. No spectral measurements are available though, so the possible contribution of SWCX is only speculative.

We know however that SWCX, and hence soft X-ray emission driven by the solar wind, takes place in the Earth's magnetic environment, particularly in the magnetosheath and the magnetospheric cusps. This was found to be the explanation of episodes of variable soft X-ray background first observed by ROSAT (Snowden et al. 1995) and subsequently by Chandra (Wargelin et al. 2004) and Suzaku (Fujimoto et al. 2007), and recently investigated in detail using XMM-Newton observations (Carter et al. 2008, 2010, 2011). What has been seen as an unwanted variable soft $X$ ray noise affecting X-ray lines-of-sight crossing magnetic field regions with high particle density is now turning into a diagnostic tool for solar-terrestrial relationships: the SMILE (Solar wind Magnetosphere Ionosphere Link Explorer) mission, a joint project by ESA and the Chinese Academy of Sciences currently under development and due for launch in 2021, will be dedicated to imaging the soft X-ray SWCX emission in the near-Earth environment, setting it into the context of solar wind conditions (Raab et al. 2016). Fig. 8 shows the result of MHD simulations by T. Sun (private communication) displaying the expected X-ray emissivity from the dayside magnetosphere following the arrival of an interplanetary shock, and gives an example of the images that a wide field-of-view X-ray telescope, such as the one which is part of the SMILE payload, can return.

\section{Discussion and conclusions}

In looking ahead to the next decade of observing with XMM-Newton it is worth considering when and how past observations have been found to return the most science. Observations at times of enhanced solar activity and comparison with those at quiet times enable us to determine what is really driving auroral and magnetospheric Xray emissions. In addition, simultaneous observations with other facilities (remote, such as HST, and in situ, such as planetary missions) enable us to put the X-ray data into context and to gather deeper insights in the underlying physics of planetary X-ray emissions.

The main current objective of X-ray exploration of the solar system is that of establishing how the solar wind interacts with planetary magnetospheres and exospheres, and comets, at different times in the solar cycle. This has far reaching implications also for other fields of research, such as exoplanets, to name the currently hottest topic. The difficulty in correlating X-ray emission to solar wind conditions resides in the uncertainty inherent to propagations from solar wind conditions at $1 \mathrm{AU}$. This is resolved if in situ measurements are available. The uniqueness of coincidence with Juno's operations at Jupiter makes the next couple of years of XMM-Newton Jovian observations invaluable. Similarly, Mars observations while MAVEN orbits the planet give insights in its outflowing exosphere under changing solar wind conditions. Finally, from the more general X-ray astronomy point of view, planetary X-ray studies offer strong synergy with attempts of establishing the SWCX contribution to the diffuse soft X-ray background.

The outlook over the next decade for solar system X-ray studies is bright: we have the potential of building substantially on the past and current research work, and of answering many of the questions it has raised, while drawing on the still excellent capabilities of XMM-Newton and Chandra and on in situ measurements such as those returned by Juno, now orbiting Jupiter.

Acknowledgements. Many thanks are due for their inputs to this review to K. Dennerl, A.Bhardwaj, D. Koutroumpa, S. Wolk and for the contributions by many others over the years to this fast growing field of research.

\section{References}

Bhardwaj, A., Elsner, R. F., Waite, J. H., Jr., Gladstone, G. R., Cravens, T. E., Ford, P. G.: 2005a, ApJ 624, L121

Bhardwaj, A., Elsner, R. F., Waite, J. H., Jr., Gladstone, G. R., Cravens, T. E., Ford, P. G.: 2005b, ApJ 627, L73

Bhardwaj, A., Gladstone, G. R., Elsner, et al.: 2007, JASTP 69, 179

Bodewits, D., Christian, D. J., Torney, M., et al.: 2007, A\&A 469, 1183

Branduardi-Raymont, G., Bhardwaj, A., Elsner, et al.: 2007, A\&A 463, 761

Branduardi-Raymont, G., Elsner, R. F., Galand, M., Grodent, D., Cravens, T. E., Ford, P., Gladstone, G. R., Waite J. H. Jr: 2008, JGR 113, A02202 

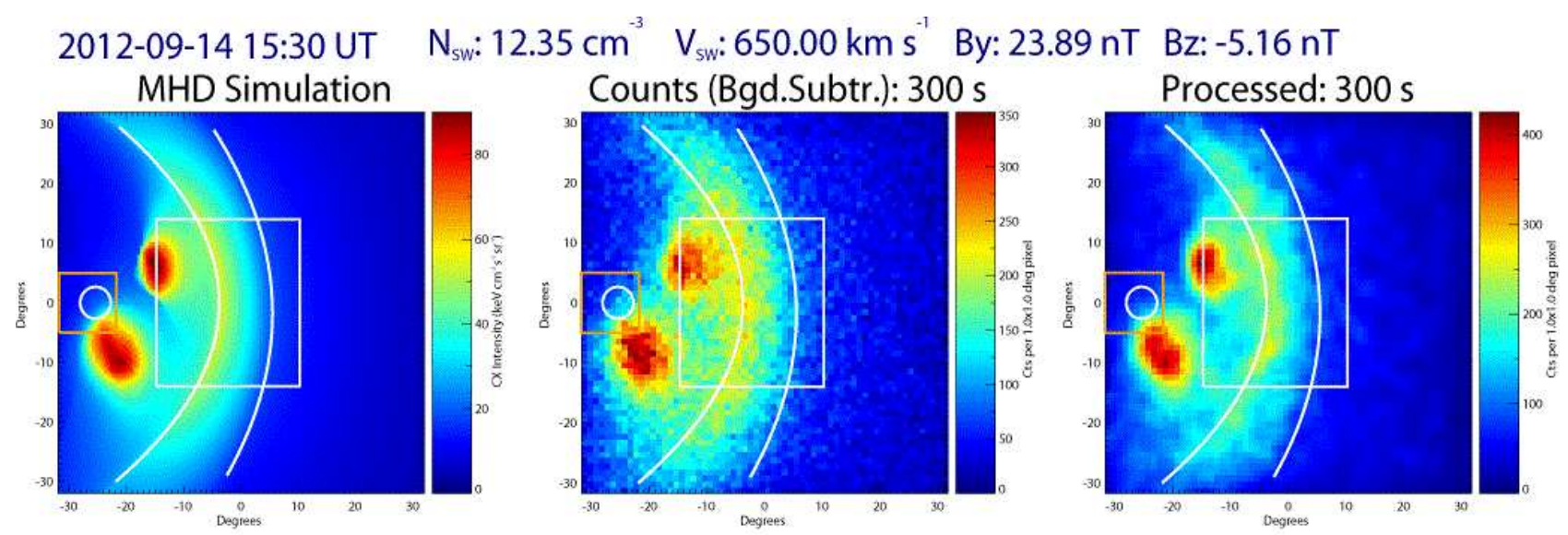

\section{Position: 8.58 5.16 17.03 GSE Aim Point: 8.48 0.00 0.00 GSE}

Fig. 8 Left: Simulated dayside magnetosphere X-ray emissivity after the arrival of an interplanetary shock. Centre: Predicted count image from a wide field-of-view X-ray telescope as the one which is part of the SMILE payload. Right: The count image after processing resulting in sharper magnetospheric boundaries (from Raab et al. 2016).

Bunce, E. J., Cowley, S. W. H., Yeoman, T. K.: 2004, JGR 109, A09S13

Carter, J. A, Sembay, S.: 2008, A\&A 489, 837

Carter, J. A, Sembay, S., Read, A. M.: 2010, MNRAS 402, 867

Carter, J. A, Sembay, S., Read, A. M.: 2011, A\&A 527, A115

Cravens, T. E.: 1997, GeoRL 24, 105

Cravens, T. E.: 2000, ApJ 532, L153

Cravens, T. E., Waite, J. H., Jr, Gombosi, T. I., Lugaz, N., Gladstone, G. R., Mauk, B. H., MacDowall, R. J.: 2003, JGR 108, 1465

Dennerl, K., Aschenbach, B., Burwitz, V., Englhauser, J. Lisse, C. M., Rodriguez-Pascual, P.: 2003, SPIE 4851, 277, in X-Ray and Gamma-Ray Telescopes and Instruments for Astronomy, J. E. Truemper, H. D. Tananbaum Ed.s

Dennerl, K., Lisse, C. M., Bhardwaj, A., et al.: 2006, A\&A 451, 709

Dennerl, K.: 2008, P\&SS 56, 1414

Dennerl, K.: 2010, SSRv 157, 57

Dunn, W. R., Branduardi-Raymont, G., Elsner, R. F., et al.: 2016, JGR 121, 2274

Elsner, R. F., Gladstone, G. R., Waite, J. H., Jr., et al.: 2002, ApJ 572, 1077

Fujimoto, R., Mitsuda, K., McCammon, D., et al.: 2007, PASJ 59, 133

Gladstone, G. R., Waite, J. H., Jr., Grodent, D. et al.: 2002, Natur 415,1000

Horanyi, M., Cravens, T. E., Waite, J. H., Jr: 1988, JGR 93, 7251

Hui, Y., Schultz, D. R., Kharchenko, V. A., Stancil, P. C., Cravens, T. E., Lisse, C. M., Dalgarno, A.: 2009, ApJ 702, L158

Hui, Y., Schultz, D. R., Kharchenko, V. A., et al.: 2010, JGR 115, A07102

Kimura, T., Kraft, R. P., Elsner, R. F., et al.: 2016, JGR 121, 2308

Lisse, C. M.; Dennerl, K.; Englhauser, J. et al.: 1996, Sci 274, 205

Metzger, A. E., Gilman, D. A., Luthey, J. L., Hurley, K. C., Schnopper, H. W., Seward, F. D., Sullivan, J. D.: 1983, JGR 88,7731

Ness, J.-U., Schmitt, J. H. M. M., Wolk, S. J., Dennerl, K., Burwitz, V.: 2004, A\&A 418, 337

Raab, W., Branduardi-Raymont, G., Wang, C., et al.: 2016, SPIE 9905, 990502, in Space Telescopes and Instrumentation 2016:
Ultraviolet to Gamma Ray, J.-W. den Herder, T. Takahashi, M. Bautz Ed.s

Snowden, S. L., Freyberg, M. J., Plucinsky, P. P., et al.: 1995, ApJ 454,643

Vogt, M. F., Kivelson, M. G., Khurana, K.K., Walker, R.J., Bonfond, B., Grodent, D., Radioti, A.: 2011, JGR 116, A03220

Waite, J. H., Jr, Clarke, J. T., Cravens, T. E., Hammond, C. M.: 1988, JGR 93, 7244

Wargelin, B. J., Markevitch, M., Juda, M., Kharchenko, V., Edgar, R.; Dalgarno, A.: 2004, ApJ 607, 5961 